\title{
Carcinoma of the appendix - A diagnostic challenge
} Bhattarai $\mathrm{S}^{1}$, Yadav BK${ }^{1}$, Kafle $\mathrm{P}^{2}$, Maharjan $\mathrm{N}^{2}$, Shrestha $\mathrm{SJ}^{2}$, Belbase $\mathrm{N}^{3}$ Kumar $\mathrm{S}^{4}$, Patowary BN ${ }^{5}$

${ }^{1}$ MBBS Intern, ${ }^{2}$ Post Graduate Resident, ${ }^{3}$ Lecturer, ${ }^{4}$ Assistant Prof., ${ }^{5}$ Prof. and HOD Department of Surgery, College of Medical Sciences and Teaching hospital, Bharatpur, Nepal.

\section{ABSTRACT}

\section{INTRODUCTION}

Primary tumors of the appendix are rare. They are usually diagnosed after pathologic inspection of the appendix removed for suspected appendicitis. The main presentation is that of an acute appendicitis or as a palpable mass, mainly in the right lower quadrant. It mimics ovarian pathology in female because of close proximity to its anatomical structures.

\section{CASE PRESENTATION}

We report a case of 52 years female who presented to us in casualty with complains of pain abdomen and other findings suggestive of acute appendicitis. In spite of extensive preoperative investigations, the diagnosis was confirmed only after opening the abdomen as giant mucocele of appendix demanding right hemicolectomy as a treatment modality.

\section{CONCLUSION}

Although primary tumors of appendix is rare identity in the arena of surgery, it should be in the corner of thought as a possible diagnosis which require extensive surgery when compared to simple Appendicectomy.

\section{Keywords: Appendicular mass, Mucocele of appendix, hemicolectomy}

\section{INTRODUCTION}

Currently, the assessment of pelvic masses relies heavily on USG as the primary diagnostic tool. However, this may not always identify the origin of such a mass. In such scenario, clinical findings and other investigative modalities are warranted to aid the diagnostic process. In spite of extensive preoperative investigations, the diagnosis may still remain elusive and may only be made at the time of surgery.1

Correspondence: Dr. Brij Kumar Yadav

Email: drbrij146@gmail.com

\section{CASE DETAIL}

Adenocarcinoma of the appendix is a rare case and we present such a case presenting in College of Medical Sciences and Teaching Hospital, Bharatpur, Chitwan, Nepal.

A 52 year post-menopausal woman was referred case from other hospital to the emergency department with right lower abdominal pain and fever on and off since last five days. She was apparently well five days back 
when she developed pain in right lower abdomen during morning time while jogging and she has to stop walking and got relieved by taking oral painkiller. It was pricking in nature, radiated towards back and associated with nausea and vomiting She gives history of significant weight loss and anorexia since six months. There was no associated urinary or bowel symptoms.

On examination, there was a cystic mass in the right iliac fossa (RIF) which was elongated, immobile and tender on touch, size about $15 \times 10 \mathrm{~cm}$. Ascites and shifting dullness were absent. Mass was not bimannually palpable per vaginally and cervical motion tenderness was not elicitated. On investigation leucocytosis was noted with neutrophilia, lymphocytosis. An ultrasound scan showed a right sided elongated cystic mass measuring $12.8 \times 6.4 \mathrm{~cm}$. The CEA value was grossly increased and CA-125 within normal limit. On further evaluation with CT scan, it revealed appendicular cystic mass suggestive of mucocele of appendix.

She was than posted for emergency laparatotomy. On opening the abdomen, there was no ascites,no peritoneal deposits. There was a huge yellowish, shiny cystic, elongated banana shaped mass in the right iliac fossa originating from caecal base suggesting giant appendicular mass which is shown in figure below. Intraoperatively appendicular lump was present in the right iliac fossa. There was no ascites, no liver nodules ,no lymph node enlarged. Pouch of Douglas was clear.She underwent right hemi colectomy with end to end ileo - transverse anastomosis.Cut section of the mass showed whitish clear gelly like material and the appendiceal orifice was indurated and hard.Orificeal diameter was $>2 \mathrm{~cm}$. Histopathology showed intermediate grade of mucinous tumor of appendix.

Post operatively patient was transferred to ICU then to general ward. Post operative periods were uneventful. Patient was discharged with smiling face and asked to follow up after 2 weeks when she presented with no any complains.

\section{Figures}

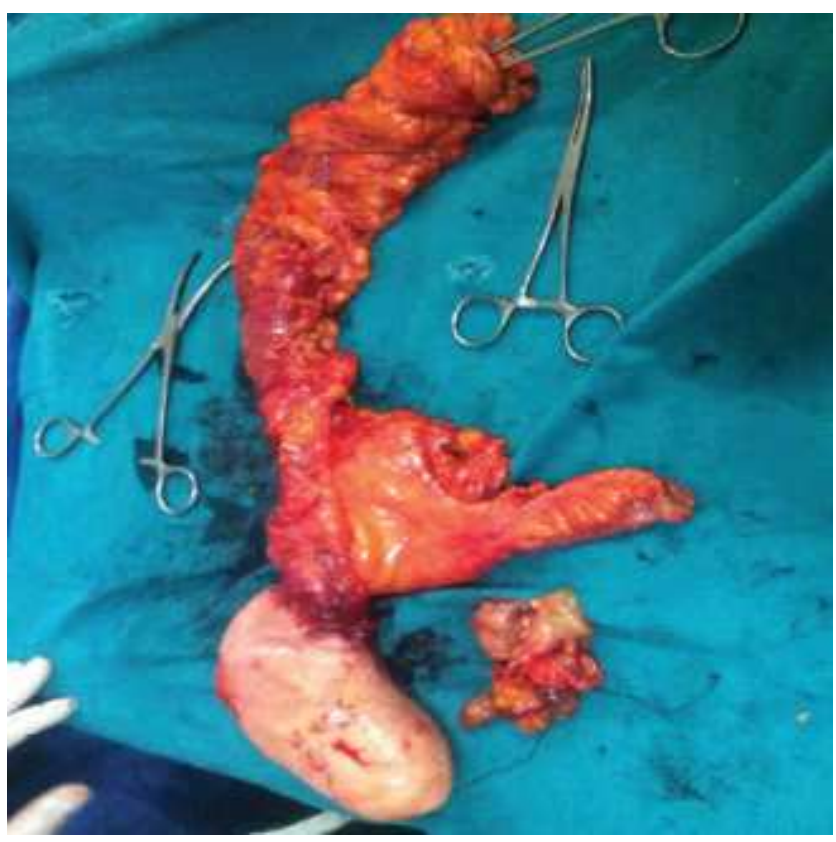

Figure 1. Resected specimen of right hemicolectomy showing appendicular mass.

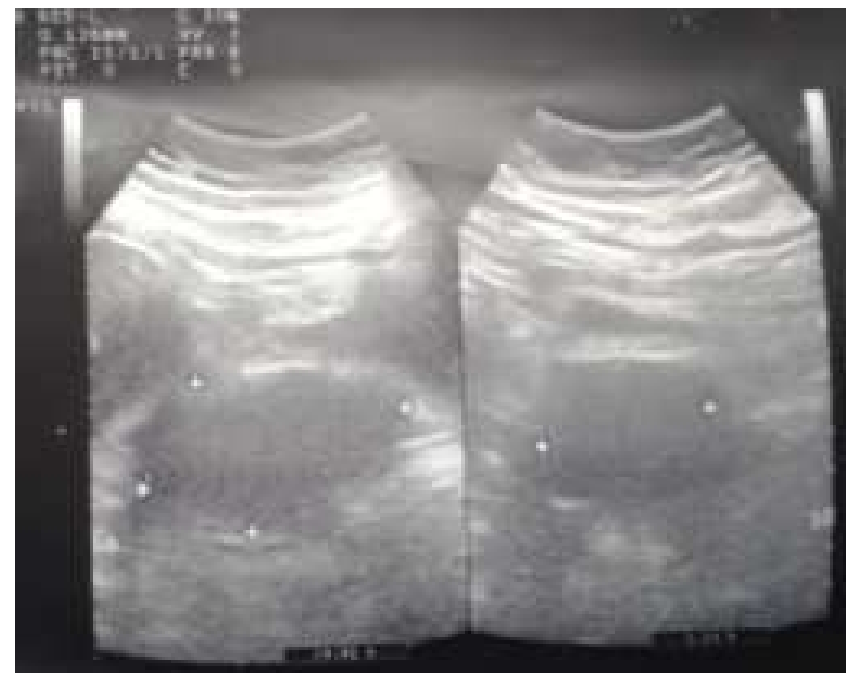

Fig 2: USG finding of appendicular cystic mass. 


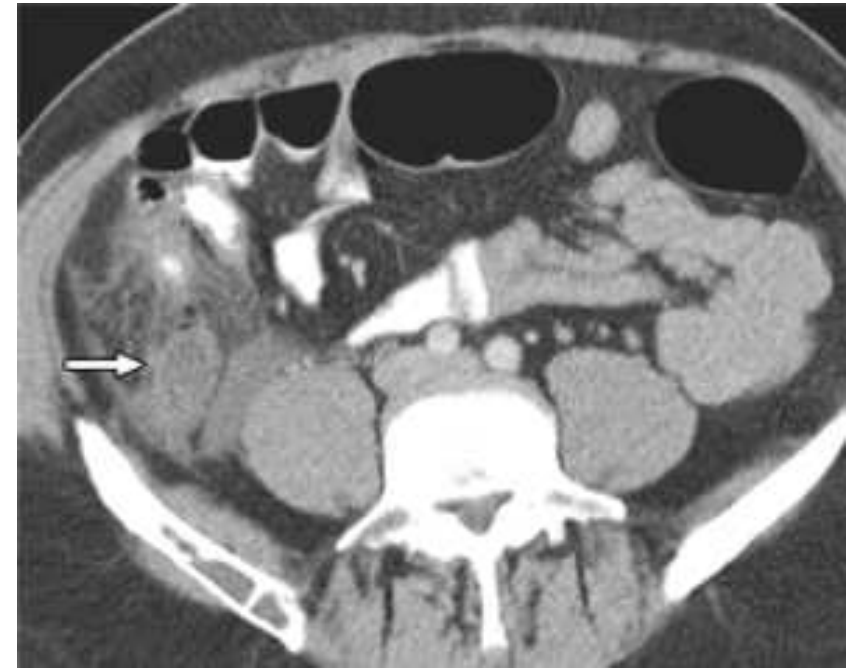

Fig 3: CT-Scan revealed cystic mass.

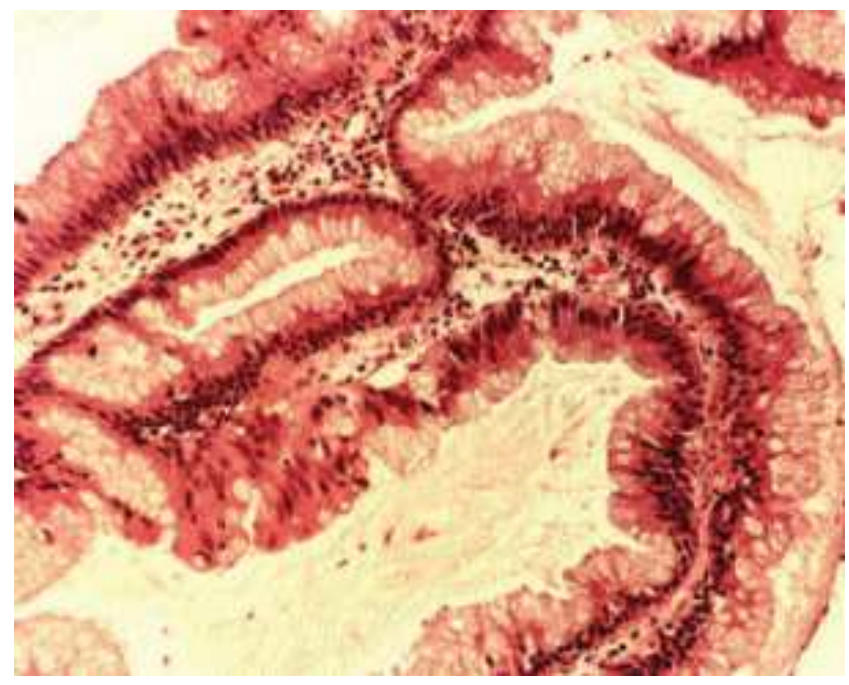

Fig. 4: Intermediate grade mucinous tumor of appendix.(H and E x 200)

\section{DISCUSSION}

Primary tumors of the appendix are rare. They are usually diagnosed after pathologic inspection of the appendix removed for suspected appendicitis. Although it was previously believed that carcinoid tumors were the most common appendiceal neoplasms, analysis of the Surveillance, Epidemiology, and End Results (SEER) database indicates that mucinous tumors of the appendix are more common. ${ }^{[2]}$
Appendiceal carcinoids are neuroendocrine tumors that are usually of the enterochromaffin cell type. They frequently contain sustentacular cells that express $S$ $100 .^{3}$ Although they are classified as malignancies, most appendiceal carcinoids exhibit benign behavior patterns. These tumors tend to occur in patients in their 40 s, and most are localized to the appendix at the time of presentation. ${ }^{2}$ For lesions smaller than $1 \mathrm{~cm}$ that are located in the tip of the appendix, appendectomy is curative nearly $100 \%$ of the time. ${ }^{2}$ Appendiceal carcinoids that are larger than 1 to $2 \mathrm{~cm}$, involve the base of the appendix, or invade the mesoappendix, may exhibit a more aggressive biologic behavior and warrant consideration of right hemicolectomy. A large population-based study showed that goblet cell histology was associated with a particularly poor prognosis among carcinoids. ${ }^{4}$ As with other neuroendocrine tumors, patients with appendiceal carcinoids may present with second primary tumors, especially of the gastrointestinal and genitourinary tracts, and this likelihood was recently estimated at $18.2 \% .^{5}$ Thus, these patients undergo colonoscopy and other screening procedures, as appropriate, based on the age of the patient and additional risk factors.

Noncarcinoid tumors of the appendix are rare tumors that typically present as appendicitis in an older patient. The duration of symptoms is usually longer. A mild anemia may be present ${ }^{6}$. however, the diagnosis is not usually made preoperatively. The mucinous adenocarcinoma cell type is most common and has a better prognosis after resection than does the colon or signet-ring cell type, with 5-year survival rates approaching 50\%. ${ }^{6}$ Some patients with mucinous tumors present with a distended appendix containing mucus, called a mucocele. Right hemicolectomy is recommended for noncarcinoid cancers larger than 1 $\mathrm{cm}$. 
A mucocele of the appendix is the result of obstruction of the appendiceal orifice with distention of the appendix caused by intraluminal accumulation of mucoid material. The mucosa of the appendix may contain benign epithelium associated with a retention cyst, mucosal hyperplasia, or low-grade atypia noted in low-grade appendiceal mucinous neoplasms. However, the epithelium may also exhibit malignant changes as with mucinous adenocarcinoma. ${ }^{[7]}$ Intact mucoceles smaller than $2 \mathrm{~cm}$ are nearly always benign. ${ }^{8}$ A retention cyst results from chronic obstruction of the proximal lumen, usually by fibrous tissue, which leads to a simple mucocele that is lined by flattened cuboidal epithelium and is cured by appendectomy. Larger mucoceles are more likely to be neoplastic. Every effort is made to keep the mucocele intact during extraction, including placing the specimen in a bag or converting a laparoscopic procedure to open if necessary. ${ }^{8}$ The mesoappendix is removed with the appendix to determine lymph node status, but a right hemicolectomy is not indicated unless there is invasion of the base of the appendix by tumor. ${ }^{[9]}$

Mucoceles that have ruptured are more likely to be associated with spread of epithelial cells in mucoid fluid throughout the peritoneal cavity, so-called pseudomyxoma peritonei, or mucinous carcinomatosis of appendiceal origin. The appendix is the most common source of mucoid fluid collections in the abdomen. Patients may present with appendicitis or in a less acute fashion with increased abdominal girth, an ovarian mass or an inguinal hernia (in men) due to intraperitoneal accumulation of mucoid material. Preoperative imaging studies may reveal enlargement of the ovaries due to entrapment of mucinous tumor cells within ovarian tissue, or focal mucinous fluid collections in the pelvis, left paracolic gutter, subhepatic space, splenic hilum, and omentum. Initially in our case we even thought a differential diagnosis of twisted ovarian cyst on USG imaging .Although nearly all patients have peritoneal dissemination of tumor cells at the time of diagnosis, most of these neoplasms are noninvasive. ${ }^{9}$ Nodal and liver metastases are uncommon, whereas locoregional recurrence of mucinous tumors, which ultimately impair small bowel function, is typical. The bulk of the peritoneal tumor is an important prognostic factor independent of histologic grade. ${ }^{7}$ An aggressive surgical approach involving extensive removal of the peritoneum and perioperative intraperitoneal chemotherapy may improve survival for these patients, especially if performed early in the course of this disease before tumor cells become trapped in scar tissue surrounding the viscera. ${ }^{9}$

\section{CONCLUSION}

Mucocele of the appendix can mimic an adnexal mass and prove to be a diagnostic challenge. In a woman presenting with right iliac fossa mass and with clinical features not indicative of gynaecological pathology, an appendiceal origin should be considered in the differential diagnosis.

\section{REFERENCE}

1. Bartlett C, Manoharan M, Jackson A, Mucocele of the appendix - a diagnostic dilemma: a case report, Journal of Medical Case Reports 2007, 1:183 doi:10.1186/1752-1947-1-183

2. McGory ML, Maggard MA, Kang H, et al. Malignancies of the appendix: beyond case series reports. Dis Colon Rectum. 2005 Dec;48(12):2264-71

3. Carr NJ, Sobin LH. Neuroendocrine tumors of the appendix. Semin Diagn Pathol 2004; 21:108-19. 
Journal of College of Medical Sciences-Nepal, 2014, Vol-10, No-2

4. McCusker ME, Cote TR, Clegg LX, et al. Primary malignant neoplasms of the appendix: A populationbased study from the surveillance, epidemiology and end-results program, 1973-1998. Cancer 2002; 94:3307-3312.

5. Modlin IM, Lye KD, Kidd M. A 5-decade analysis of 13,715 carcinoid tumors. Cancer 2003; 97:934-959.

6. Todd RD, Sarosi GA, Nwariaku F, et al. Incidence and predictors of appendiceal tumors in elderly males presenting with signs and symptoms of acute appendicitis. Am J Surg 2004; 188:500-504.

7. Misdraji J, Yantiss RK, Graeme-Cook FM, et al. Appendiceal mucinous neoplasms: A clinicopathologic analysis of 107 cases. Am J Surg Pathol 2003; 27:1089-1103.

8. Dhage-Ivatury S, Sugarbaker PH. Update on the surgical approach to mucocele of the appendix. $J$ Am Coll Surg 2006; 202:680-684. 